\title{
EFFICIENT MONITORING OF TIME SERIES DATA USING DYNAMIC ALERTING
}

\author{
By \\ GIRISH L. * \\ DEEPTHI T. K. ** \\ * Assistant Professor, Department of Computer Science and Engineering, Channabasaveshwara Institute of Technology, \\ Gubbi, Karnataka, India. \\ ** PG Student, Department of Computer Science and Engineering, Channabasaveshwara Institute of Technology,
} Gubbi, Karnataka, India.

Date Received: 04/09/2018

Date Revised: $27 / 09 / 2018$

Date Accepted: 17/11/2018

\section{ABSTRACT}

Network and Cloud Data Centers generate a lot of data every second, this data can be collected as a time series data. A time series is a sequence taken at successive equally spaced points in time, that means at a particular time interval to a specific time, the values of specific data that was taken is known as a data of a time series. This time series data can be collected using system metrics like CPU, Memory, and Disk utilization. The TICK Stack is an acronym for a platform of open source tools built to make collection, storage, graphing, and alerting on time series data incredibly easy. As a data collector, the authors are using both Telegraf and Collectd, for storing and analyzing data and the time series database InfluxDB. For plotting and visualizing, they use Chronograf along with Grafana. Kapacitor is used for alert refinement and once system metrics usage exceeds the specified threshold, the alert is generated and sends it to the system admin.

Keywords: Time Series Data, Influxdb, Alerts, Thersholding.

\section{INTRODUCTION}

Monitoring the system is most important in the modern datacenter applications because it helps you find many issues before they cause any major downtime that affect your end users and may damage your complete business reputation. Also, it helps you to plan growth based on the real usage of your system. Collection of different metrics from many data sources is not enough, still we need to personalize our system monitoring to meet your own business needs and define the right alerts so that any abnormal changes in the system will reported (Rashmi et al., 2015).

Network and Data centers generate a lot more data per day. This can be called as Time series Data. Time series is a set of data depending upon time with observations taken at specified time. It may be defined as a collection of reading belonging to the different time periods (Time Series, n.d.). The data metrics retrieved by variable of time is called as Time Series (Collectd, n.d.). A particular time interval, the values of specific data were taken, which known as the data of time series. This time series data can be collected using system metrics like CPU, Memory, or Disk. For a given set of time intervals, the observations reproduced are said to be Discrete time series (Grafana, n.d.). If some time interval observations are read incessantly then it is known as Continuous-time series.

Figure 1 shows indication of the CPU idle time for a particular time interval with respect to values. The major time-serial publication-related tasks contain question by data known as anomaly detection (Chhetri et al., 2016). Time-series data processing reveals frequent sides of

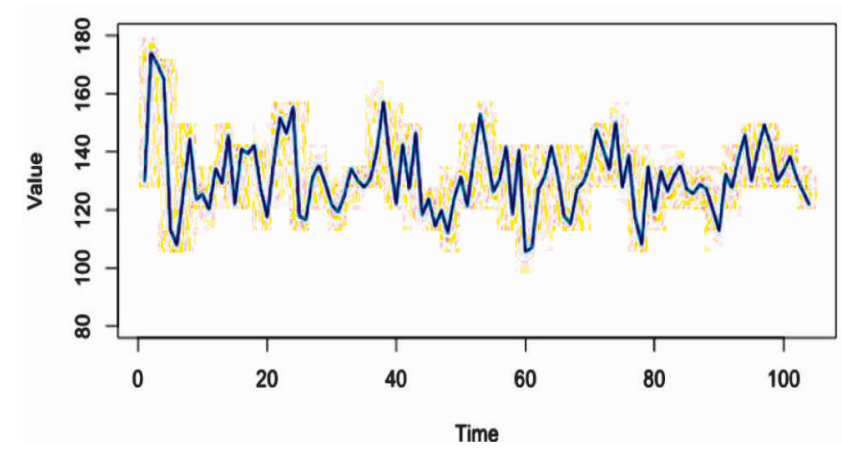

Figure 1. Time Series Data of CPU Time 


\section{RESEARCH PAPERS}

quality. The foremost vital issues are similarity (Khanum \& Girish, 2015) measures, data representations, and classification (Thara \& Girish, 2015) ways. This paper went over a number of the time-series data mining (Rashmi et al., 2015) classifications (Thara et al., 2016; Nayana et al., 2015) and offers the information of data based clusters.

To analyze the past behavior of a variable in order to predict its future behaviour, the authors use time series analysis. In the network system, the performance or the failure of the system depends on the different system metrics like load and the packet loss due to the transmission. In such cases, it is difficult to analyse or understand the system failure in advance.

The main objectives of this paper is to review the different monitoring solutions for network, to develop a different alert based on the system metrics for CPU load, to send an alert message to the system, and the network administrators rejecting the failure of the system. By using dynamic thresholding, we can send an alert message to the authorized person of an organization or data centre when the thresholding value has been exceeded. For implementation of dynamic thersholding, the authors have used TICK stack components like Telegraf (What is Telegraph, n.d.), InfluxDB (The Time Series Database in the TICKStack, n.d.), Chronograf (Grafana, n.d.), and Kapacitor. For collecting the metrics, they use Telegraf, for storing the authors use InfluxDB, for plotting and visualizing they use Chronograf and for data processing they use Kapacitor.

Once the data is collected from Collectd and Telegraf, different types of analysis can be done. Chronograf is the visualization part of Influx Data's TICK Stack. It makes the monitoring and alerting for your infrastructure easy to setup and stack. It is easy to manage and includes templates with various libraries to allow you to rapidly create dashboards of your data with real-time visualizations. It is an algorithm based on conformance threshold that is dynamically defined (Girish \& Rao, 2016). The algorithm was evaluated on two datasets of artificial logs (one with 360 complex logs and other with 1800 simpler logs) (Khanum \& Girish, 2015). One cannot manage a fast-growing system without a deeper understanding of how your applications are working.
When considering a monitoring system, the hard part is that it needs to be up when all the other systems are down. If your monitoring goes down with your infrastructure (TICKstack, n.d.), then you will have an even bigger problem. That is why one needs the best tools and methodology to manage the applications on own (Thara \& Girish, 2015).

\section{Overview of TICK Stack}

\subsection{Data Collection}

An agent is used for collecting and reporting metric and event. It is a part of TICK stack and is a plugin-driven server agent for collecting and reporting metrics. Telegraf has a combination of plugins and rules for retrieving the metrics from the various system metrics. It takes different metrics from Application Program Interface (API) or it will listen for metrics via StatsD and Kafka consumer services (TICKstack, n.d.). Also, Telegraf has output plugins for sending the collected metrics data to different data stores like influxdb, grphite, opentsdb, and kafka.

\subsection{Time Series Database}

InfluxDB is used as a data store for many use cases consisting of large amounts of time stamped data and also used in many application metrics monitoring and real time analytics (Assfalg et al., 2006). It can be possible to run influxdb on the machine by configuring to defined length of time. Automatic expiring and deleting any unwanted data from the system is carried out by setting the trash conditions. In constrant to that InfluxDB provides an interaction with the data in the form of SQL-like (Thara \& Girish, 2015).

\subsection{Visualization}

Chronograf is the user interface component of Influx Data's TICK Stack as shown in Figure 2. It helps in monitoring and alerting the infrastructure by simplifying the setup and maintenance tasks (Rashmi et al., 2015).

\section{Literature Survey}

Johannes Assfalg et al. (Assfalg et al., 2006) have used the concept of adaptable threshold similarities for analysing time series. Theoretical issue of information mining in time arrangement databases is of most extreme significance for some practical applications and has been fixed to a lot of research in the past years. In this paper, the authors 


\section{RESEARCH PAPERS}

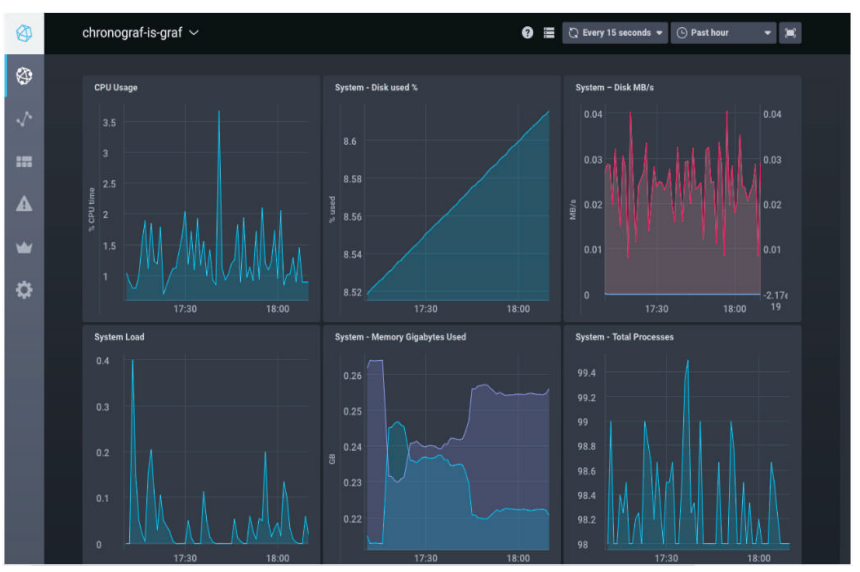

Figure 2. The System Resources View in Chronograf (Source: http://filmi-onlain.info/jpgcpng-chronograf-influx.shtml)

have centered around the as of late proposed idea of edge likeness, which thinks about the time arrangement in view of the time allotments inside which they surpass a user- defined amplitude threshold.

Fabio Bezerra and Woiner use a dynamic threshold algorithm for anomaly detection. In the recent years, organizations have adhered to Process Aware Information Systems (PAIS) for provisioning the control of their organizations. Be that as it might while institutionalizing PAIS may exchange off the power of these associations, where PAIS has a danger for security.

To realter the trade they presented, another approach for irregularity acknowledgment in logs of PAIS was established. It is one of the calculations in the perspective of edge that is characterized progressively. They carried out work by conducting survey on two datasets of fake logs, one consisting of 360 complex logs and other with 1800 less demanding logs with different profiles. By utilizing this approach, we can complete the report for abnormality location that imprints as inconsistence in logs.

\section{System Analysis}

In previous days, the threshold value for detection process should be set manually, for which a lot of problems in system metrics like loss of system application are to be forced making the system or the application slow.

To overcome the drawbacks of the existing system, the authors have chosen the TICK (Telegraph, InfluxDB, Chronograf, kapacitor) training framework for dynamic alert refinement. The easy to use time series database using InfluxDB has chances of losing data or application. One can send alert to mail, log file, etc. Some of the advantages are increased data privacy for collected metrics, ability to tailor anomaly detection queries for metrics represented by time series with kapacitor included in InfluxData TICK stack, flexibility to analyse metrics using prediction algorithms supported by Kapacitor, and multiple alert notification channels.

\section{Proposed System}

\subsection{System Architecture}

Figure 3 describes the system architecture. Telegraf is an agent for collecting and reporting the metrics from the system. These collected metrics are in the form of time series and they are stored in time series database known as influxDB.

Kapacitor, being a real-time data processing engine can process both stream and batch data InfluxDB (Girish \& Rao, 2016). We can plug in our own custom logic or userdefined functions to process alerts with dynamic thresholds, match metrics for patterns, compute statistical anomalies, and perform specific actions based on these alerts like dynamic load rebalancing.

Chronograf acts as a visualization component in TICK Stack and it provides general API to expose the data. Chronograf makes the job of monitoring and alerting in cloud data center environment easier. It has different templates and libraries for setting up the visulaization dashboard. It also provides a rapid dashboard development with real-time visualizations of your data.

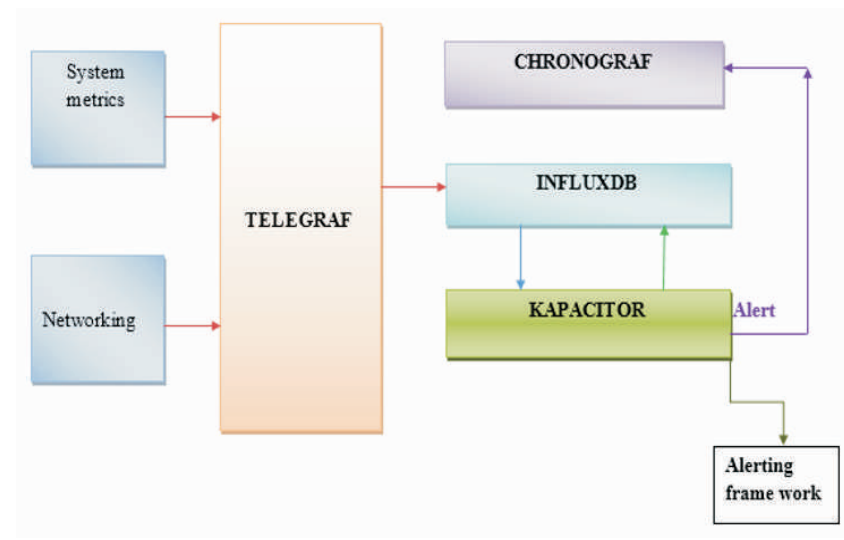

Figure 3. System Architecture 


\section{RESEARCH PAPERS}

\subsection{Flow Diagram}

Flow diagram also called as flow chart is a diagram that gives step by step actions or set of dynamic relationships between the components in a system. Flow diagrams are used to represent the structure and order for a compound system and reveal the underlying structure of the elements and their interaction.

In the below flow diagram shown in Figure 4, Telegraf calculate the system metrics like network, memory, disk, etc., from the system and stores that data in a database called influxDb. This data is further given to a data processing engine called kapacitor, it processes the data and retrieves back to the database. If the value exceeds threshold value then it gives an alert, which can be viewed in chronograf.

\section{Implementation}

For time series data management and monitoring, open source tools are widely used. In this work, the authors have used the open source tools like Telegraf, Chronograf,
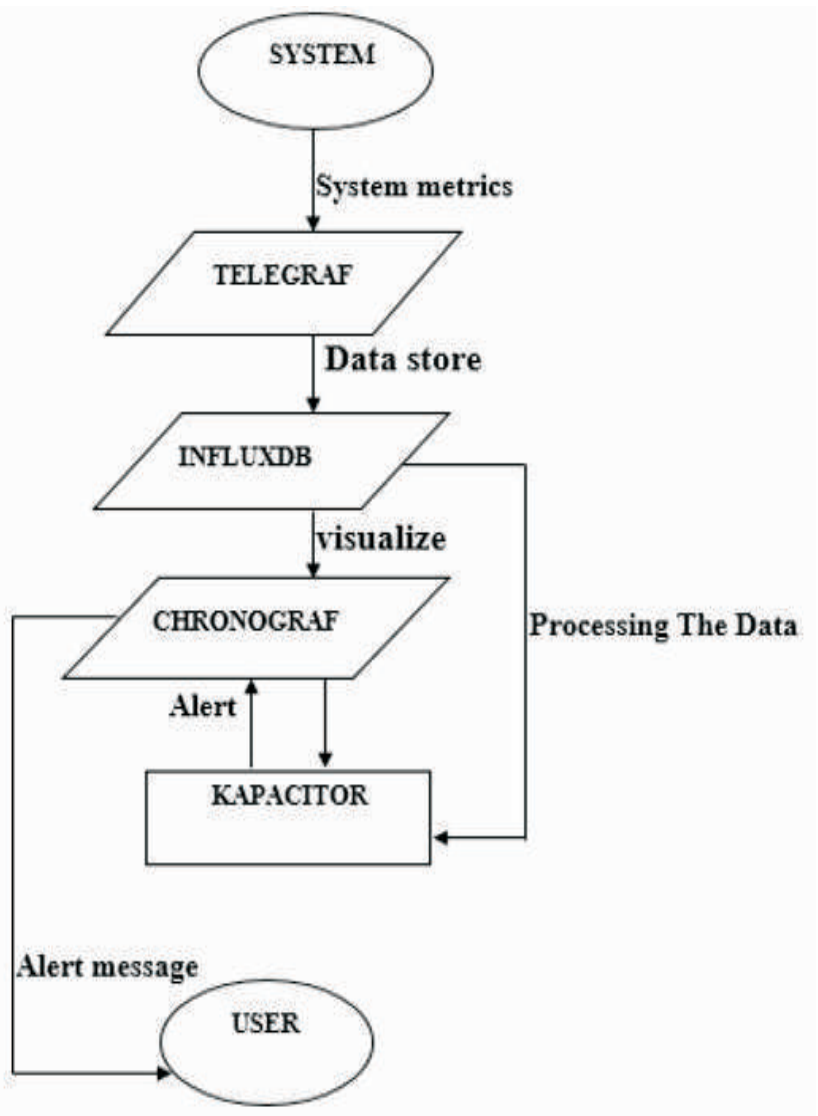

Figure 4. Flow Diagram of the Proposed System
Kapacitor, and Influxdb, altogether form an stack called as TICK. Dynamic thresholding is used for monitoring and managing the time series data.

For configuring kapacitor event handler, chronograf and kapacitor work together to send alert messages to supported event handlers. We can also use Chronograf to send alert messages to particular URLS as well as to applications like Slack.

Configuring alert kapacitor acts as a intrinsic data processing engine in the TICK Stack. It processes both stream and batch data from InfluxDB (Chhetri et al., 2016; Girish \& Rao, 2016) and allows us to plugin our own custom logic or user-defined functions to process alerts with dynamic threshold. Also it triggers an alert based on dynamic criteria. Data is stored in the InfluxDB. TICK Script language is a dynamic language that is used to Define Directed Acyclic Graphs (DAGs) (Grafana, n.d.).

Slack is a messaging app for teams. Configuring Chronograf can send alert to an existing Slack channel or as a Direct Message (DM).

Here, the alert messages will be sent by chronograf to slack channel that already exists. The channel or DM specified in the alert rule takes precedence over both the slack channel configuration option and the WebHook URL configuration. Figure 5 describes the slack channel, which use TICK stack, where Telegraf acts as an agent for collecting and sending data to InfluxDB, InfluxDB acts as a database for storing the data and sending data to chronograf, Chronograf acts as an user interface for visualizing, alert management, and Kapacitor handles alerting features in the background.

Example Stream

$$
\begin{aligned}
& \text { | from () } \\
& \text {. measurement ('mem') } \\
& \text { | alert () } \\
& \text {. crit(lambda: ("free") < 50000000) } \\
& \text {. log ('/tmp/alerts.log') }
\end{aligned}
$$

The above sample TICK Script uses stream node which is also known as initial node. It takes in streams of data from particular measurements and performs various 


\section{RESEARCH PAPERS}

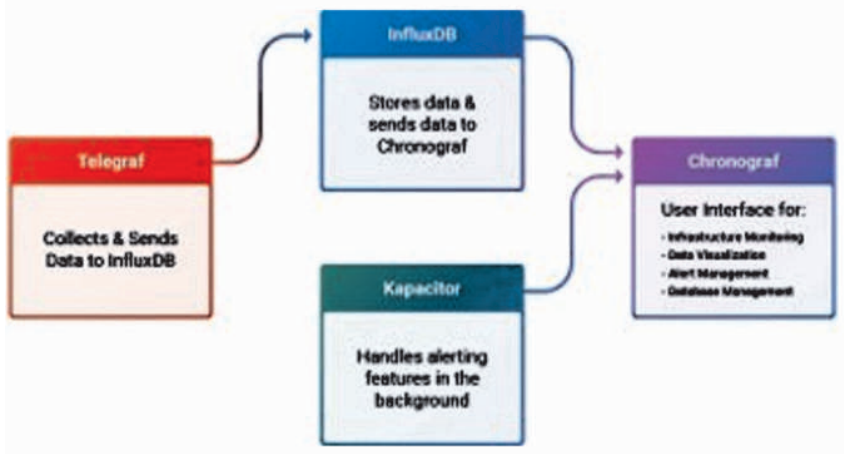

Figure 5. Slack Channel

operation. That stream of data from the measurements 'mem' issues a critical alert if the storage space is less than $500 \mathrm{MB}$.

Figure 6 describes the result for given rule condition, where alert type is a threshold that sends the alert where usage_idle is less than 80 . This can be viewed in alert end point like Slack.

\section{Conclusion}

In this paper, the dynamic alert for efficient management of time series data is implemented by using TICK stack. Once the usage of the system metrics crosses the specified threshold then alerts will be sent to the system admins through email. System admin can take actions regarding the alert. The TICK is the combination of system management tools like telegraf, influxDB, chronograf, and kapacitor. All of them offer API to build our own custom implementation. The Telegraf collects system metrics and gives to the InfluxDB to store the collected data, and to visualize the data in graphical form, we use chronograf. Automated dynamic alert is sent to the specific administrator by using TICK stack. So, it is easy for the

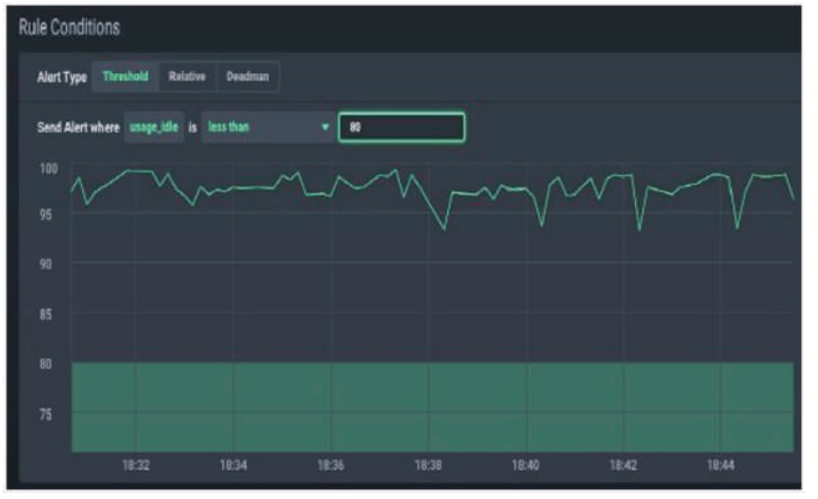

Figure 6. Rule Condition network (system) management in managing the whole network.

\section{References}

[1]. Assfalg, J., Kriegel, H. P., Kroger, P., Kunath, P., Pryakhin, A., \& Renz, M. (2006, July). Time series analysis using the concept of adaptable threshold similarity. In Scientific and Statistical Database Management, 2006. 18 International Conference on (pp. 251-260). IEEE.

[2]. Bezerra, F. D. L., \& Wainer, J. (2012). A dynamic threshold algorithm for anomaly detection in logs of process aware systems. Journal of Information and Data Management, 3(3), 316-331.

[3]. Chhetri, M. B., Vo, Q. B., \& Kowalczyk, R. (2016, December). CL-SLAM: Cross-layer SLA monitoring framework for cloud service-based applications. In Utility and Cloud Computing (UCC), 2016 IEEE/ACM $9^{\text {th }}$ International Conference on (pp. 30-36). IEEE

[4]. Collectd. (n.d.). In OPNFV. Retrieved from https://wiki. opnfv.org/display/fastpath/Collectd + 101

[5]. Girish, L., \& Rao, S. K. (2016, December). Mathematical tools and methods for analysis of SDN: A comprehensive survey. In Contemporary Computing and Informatics (IC3I), $20162^{\text {nd }}$ International Conference on (pp. 718-724). IEEE.

[6]. Grafana. (n.d.). In Archlinux. Retrieved from https://wiki.archlinux.org/index.php/Grafana

[7]. Khanum, S., \& Girish, L. (May 2015). Meta heuristic approach for task scheduling in cloud datacenter for optimum performance. International Journal of Advanced Research in Computer Engineering \& Technology (IJARCET), 4(5), 2070-2074.

[8]. Nayana, Y., Gopinath, J., \& Girish, L. (2015). DDoS mitigation using Software Defined Network. International Journal of Engineering Trends and Technology (IJETT), 24(5), 258-264.

[9]. Rashmi, T. V., Prasanna, M. K., \& Girish, L. (2015). Load balancing as a service in Openstack-Liberty. International Journal of Scientific \& Technology Research, 4(8), 70-73.

[10]. Time Series. (n.d.). In Wikipedia. Retrieved from https://en.wikipedia.org/wiki/Time_series 


\section{RESEARCH PAPERS}

[11]. The Time Series Database in the TICK Stack. (n.d.). In InfluxDB. Retrieved from https://www.influxdata. com/time- series-platform/influxdb/

[12]. TICK-stack. (n.d.). In Codeship. Retrieved from https://blog.codeship.com/infrastructure-monitoring with-tick-stack/

[13]. Thara, D. K., Premasudha, B. G., Ram, V. R., \& Suma, R. (2016, December). Impact of big data in healthcare: A survey. In Contemporary Computing and Informatics
(IC3I), $20162^{\text {nd }}$ International Conference on (pp. 729735). IEEE

[14]. Thara, D. K., \& Girish, L. (May, 2015). Efficient virtual machine memory transfer in datacenter with optimal downtime. International Journal of Engineering Trends and Technology, 23(9), 454-458.

[15]. What is Telegraph. (n.d.). In Influxdata. Retrieved from https://support.influxdb.com/hc/en-us/articles/ 212832517

\section{ABOUT THE AUTHORS}

Girish $L$ is working as an Assistant Professor in the Department of Computer Science and Engineering in Channabasaveshwara Institute of Technology, Karnataka, India. His areas of research including Cloud Computing, Machine Learning and SoftwareDefined Networking.

Deepthi T. K. is a PG Student in the Department of Computer Science and Engineering at Channabasaveshwara Institute of Technology, Karnataka, India. Her areas of research include Cloud Computing and Computer Networking.
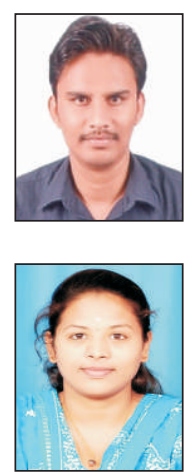